\title{
Thai national guidelines for the prevention of mother- to-child transmission of HIV: March 2010
}

\author{
Nittaya Phanuphak ${ }^{\mathrm{a}}$, Rangsima Lolekha ${ }^{\mathrm{b}}$, Kulkanya Chokephaibulkitc ${ }^{\mathrm{c}}$ Nipunporn Voramongkold, \\ Sarawut Boonsuk ${ }^{\mathrm{e}}$, Aram Limtrakul ${ }^{\mathrm{f}}$, Piyawan Limpanyalert ${ }^{\mathrm{g}}$, Sanchai Chasombat ${ }^{\mathrm{h}}$, Sombat Thanprasertsuki, \\ Manoon Leechawengwong, for the Thai National HIV Guidelines Working Group \\ ${ }^{a}$ The Thai Red Cross AIDS Research Centre, Bangkok 10330; ${ }^{b}$ Global AIDS Program, Thailand MOPH- \\ U.S. CDC Collaboration, Nonthaburi 11000; 'Department of Pediatrics, Faculty of Medicine Siriraj \\ Hospital, Mahidol University, Bangkok 10400; ' Maternal and Child Health Group, Bureau of Health \\ Promotion, Department of Health, Ministry of Public Health, Nonthaburi 11000; ${ }^{e}$ Benjalak Hospital, \\ Srisaket 33110; 'Nakornping Hospital, Chiang Mai 50180; ${ }^{9}$ Bamrasnaradura Infectious Disease \\ Institute, Ministry of Public Health, Nonthaburi 11000; ${ }^{h}$ Bureau of AIDS, TB, and STIs, Department \\ of Disease Control, Ministry of Public Health, Nonthaburi 11000; iWorld Health Organization, Thailand \\ Office, Bangkok 11000; 'Thai AIDS Society, Bangkok 10330, Thailand
}

Thailand has been one of the leading developing countries to implement a national program to prevent mother-to-child transmission (MTCT) of HIV. Although the recent transmission rate has been low, the goal is to eliminate MTCT altogether. The Thai National HIV Guidelines Working Group issued treatment guidelines to prevent MTCT in Thailand in March 2010. These guidelines will be implemented nationwide within a year. The most important aspects of these new guidelines are as follows:

Treatment in HIV-infected pregnant women who have not been on antiretroviral treatment prior to pregnancy. Antepartum treatment is recommended for all pregnant women regardless of CD4 count with highly active antiretroviral therapy (HAART) containing zidovudine (AZT) + lamivudine (3TC) + lopinavir/ritonavir (LPV/r). Treatment should be started immediately irrespective of gestational age in women with CD4 count $\leq 350$ cells/ $\mathrm{mm}^{3}$, and as early as 14 weeks of gestation in those with CD4 count $>350$ cells $/ \mathrm{mm}^{3}$. After delivery, women with baseline CD4 count $\leq 350$ cells $/ \mathrm{mm}^{3}$ are referred for long-term care and HAART according to the National Adult HIV Treatment and Care Guidelines 2010. Women with CD4 count $>350$ cells $/ \mathrm{mm}^{3}$ do not need HAART and can stop all drugs after delivery. The treatment in infants includes AZT syrup for four weeks and exclusive formula feeding.

Treatment in HIV-infected pregnant women who conceive while on HAART. Women who are stable on HAART should continue the treatment during the whole period of pregnancy. Those who are taking efavirenz (EFV) and present during the first trimester should have EFV switched to another drug. Whenever possible, AZT should be used during pregnancy. Treatment in infants is similar to the above scenario.

Treatment in women who present in labor without antenatal care. Single-dose nevirapine (SD-NVP) 200 mg must be given immediately along with oral AZT $300 \mathrm{mg}$ every three hours until delivery, or oral AZT 600 mg given as a single dose. The tail therapy of AZT $+3 T C+L P V / r$ for four weeks should be given unless these women have a CD4 count of $\leq 350$ cells/ $\mathrm{mm}^{3}$ and therefore require life-long HAART. SD-NVP should not be given if the women are to deliver within two hours. The infants in this situation should receive AZT + 3TC + NVP for four weeks.

Treatment during delivery and mode of delivery. During labor, oral AZT 300 mg every three hours or oral AZT $600 \mathrm{mg}$ given as a single dose is recommended regardless of antepartum antiretroviral (ARV) regimen or the woman's history of AZT resistance. Elective caesarean section is suggested in women who did not receive HAART (including those without antenatal care), received HAART for less than four weeks prior to delivery, had poor adherence, or had incomplete viral suppression at 36 weeks of gestation.

Keywords: HIV, mother-to-child transmission, Thai guidelines

Mother-to-child transmission (MTCT) has been the principle route of HIV infection in children, occurring in $20-40 \%$ without preventive measures. Since the first perinatal HIV infection case reported in Thailand in 1988, the number of perinatal HIV infection had increased significantly. The Thailand Ministry of Public Health (MOPH) began implementing a national program for prevention of 
mother-to-child transmission of HIV (PMTCT) for all pregnant women in 2000. The program consisted of routine voluntary HIV testing and counseling for all pregnant women, antiretroviral (ARV) prophylaxis for all HIV-infected pregnant women and their infants, and provision of infant formula. HIV-infected postpartum women and their families also received continuous long-term care [1]. Antepartum ARV drug used at the beginning of the program was zidovudine (AZT) monotherapy provided from 34 weeks of gestation. The regimen was changed in 2004 to AZT provided from 28 weeks of gestation plus single-dose nevirapine (SD-NVP) given during labor to the women and after delivery to their infants. Since 2006, MOPH has provided highly active antiretroviral therapy (HAART), primarily AZT + lamivudine (3TC) + nevirapine (NVP), to all pregnant women with CD4 count $\leq 200$ cells $/ \mathrm{mm}^{3}$. The addition of 7-day AZT + 3TC tail therapy after delivery has also been implemented in women who receive SD-NVP to reduce non-nucleoside reverse transcriptase inhibitor (NNRTI) resistance based on the results from the Tops study [2].

In 2008, the median prevalence of HIV infection in pregnant women was $0.72 \%$ [3]. From the national PMTCT evaluation program in 2007, the rate of MTCT of HIV was 2.8\% from laboratory diagnosis and was 5.6\% when including infants' deaths [4]. One of the hurdles of the intervention was that $56 \%$ of HIV-infected women did not have CD4 count measured during pregnancy, and therefore missed the chance of getting appropriate ARV regimens. Moreover, only half of the infants had the diagnostic test performed, and therefore the effectiveness of the intervention program could not be accurately evaluated. Despite the very high overall rate (95\%) of access to antenatal care in Thailand [5], only 39\% of Thai pregnant women sought care before 12 weeks of gestation [6]. The rate of antenatal care among Thai HIV-infected pregnant women, however, was lower (87\%) than pregnant women in general [4]. The median gestational age at the first antenatal care among HIV-infected pregnant women was 19 weeks, and almost a quarter presented for care after 28 weeks of gestation [7]. Of all HIV-infected pregnant women, about half had CD4 count $>350$ cells $/ \mathrm{mm}^{3}$ [4].

The World Health Organization (WHO) has recently issued the rapid advice on the use of antiretroviral drugs for treating pregnant women and preventing HIV infection in infants with the goal to increase survival of mothers and infants and finally to eliminate MTCT [8]. This new recommendation aims toward more effective ARV regimens. The regimens need to be practical and cause few undesirable side effects. Several studies showed that although AZT + SD-NVP regimen is effective [9], the subsequent development of NNRTI resistance has been a major concern for long-term treatment in postpartum women and HIV-infected infants. Moreover, HAART has been shown to be more effective for PMTCT [10] and could lead to the goal of MTCT elimination while also avoiding the problem of NNRTI resistance.

In early 2010, the Thailand National Health Security Office advised using HAART for all HIVinfected pregnant women, after the Health Intervention and Technology Assessment Program revealed a better cost benefit analysis with the use of HAART over AZT + SD-NVP in HIV-infected pregnant women with CD4 count $>250$ cells $/ \mathrm{mm}^{3}$ [11]. The implementation of HAART for all HIV-infected pregnant women was also shown to be feasible and acceptable in the field [11]. The Thailand MOPH started to implement the use of HAART for all HIVinfected pregnant women in 2010 with the plan for a countrywide coverage by the year 2011. The guidelines presented here elaborate the whole package of Thailand's new PMTCT program with the principle of providing HAART for all HIV-infected pregnant women.

\section{Use of antiretroviral drugs for the prevention of mother-to-child transmission of HIV}

HIV-infected pregnant women along with their male partners (if women's HIV status has already been disclosed to them) should be informed about the benefits of taking ARV drugs for PMTCT, side effects of ARV drugs, importance of adherence to drugs, and the fact that HIV transmission to their infants can possibly occur despite ARV use. Pregnant women should be assessed for symptoms, disease staging, CD4 count, and other baseline laboratory findings. This information will be used to guide the appropriate selection of an ARV regimen for each pregnant woman. The use of ARV regimen will be most effective if it is started very early in pregnancy along with the provision of intrapartum ARV drug to women, ARV drug(s) to infants, and adherence support. Table 1 presents the recommendations for the use of ARV drugs for PMTCT. 


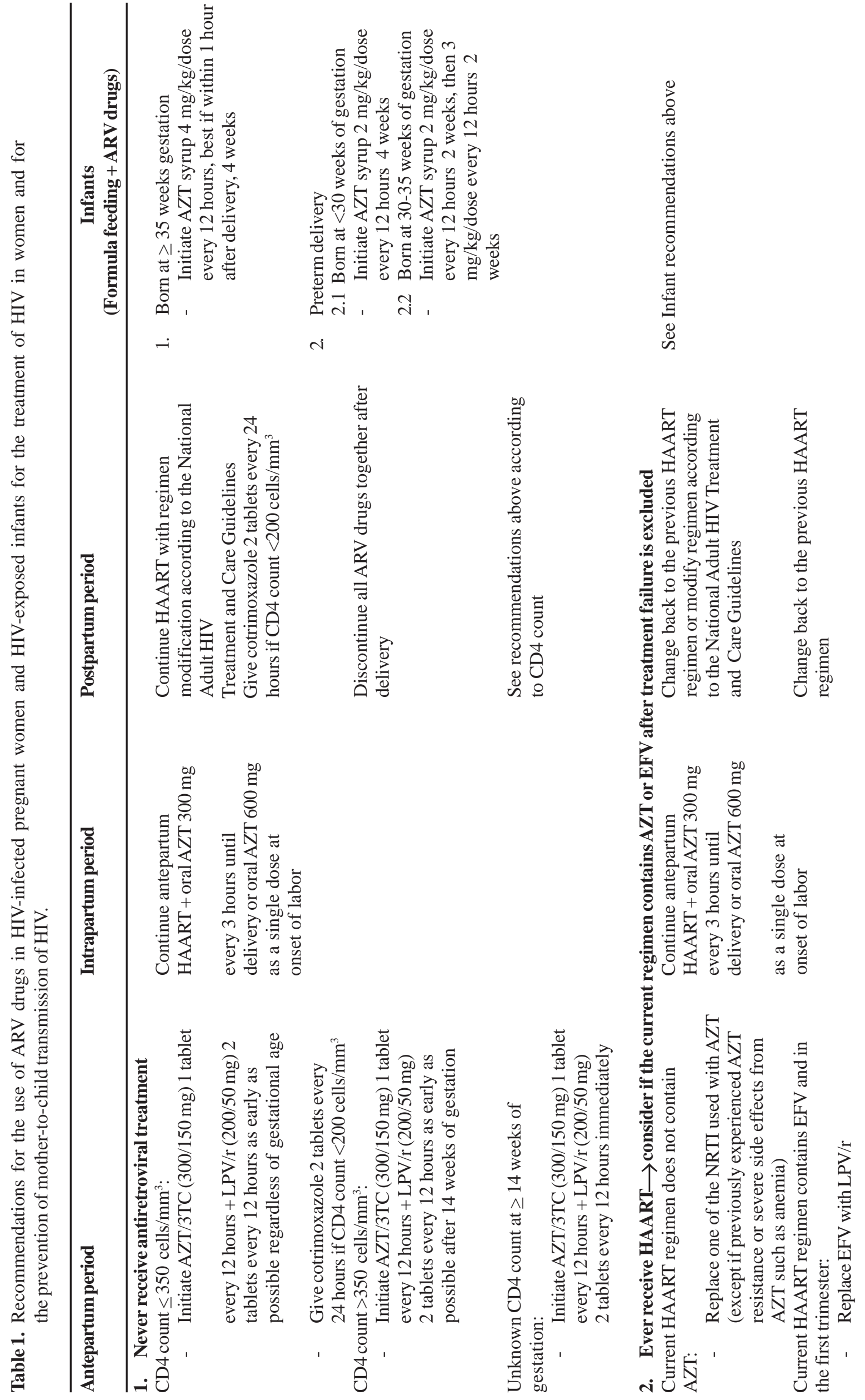




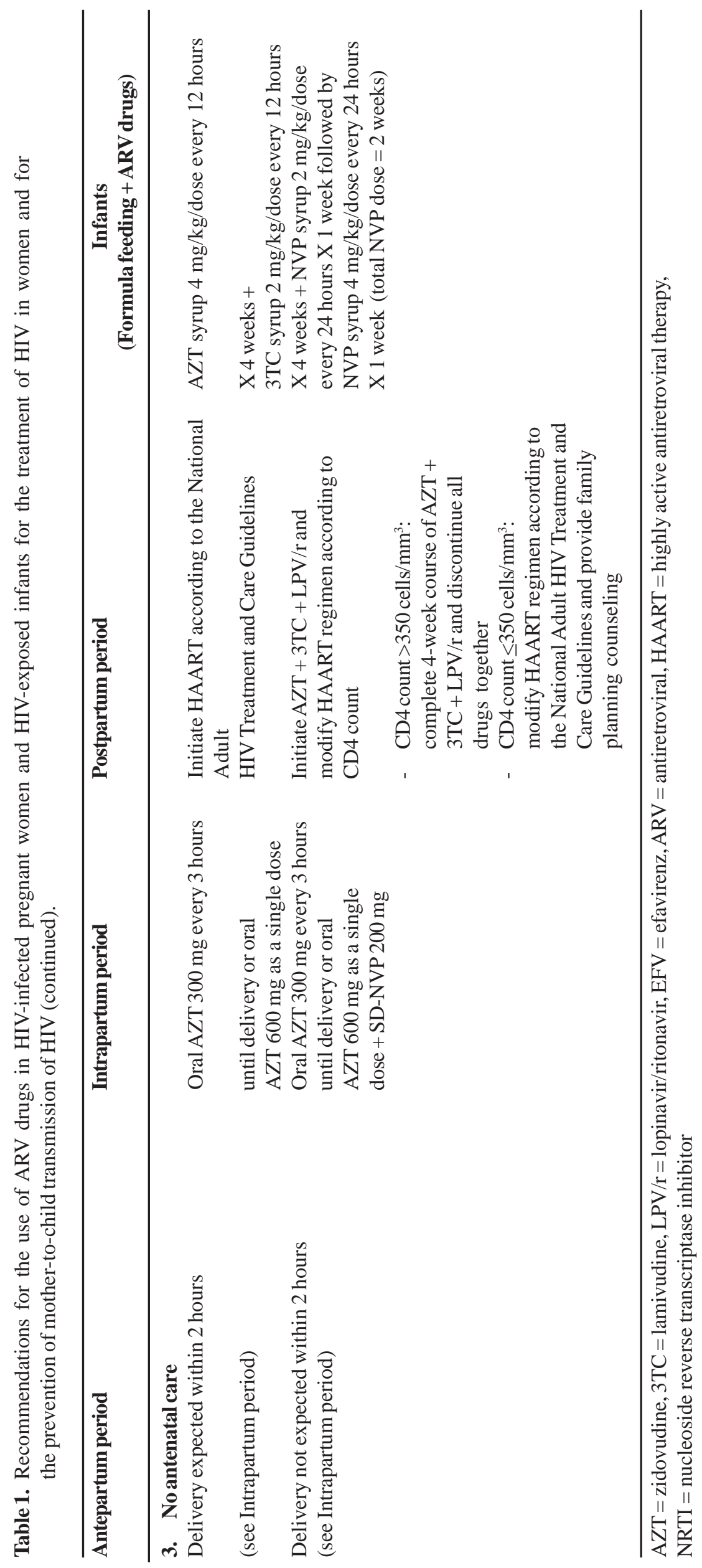




\section{Antepartum antiretroviral drugs}

Scenario 1: HIV-infected pregnant women who have not been on antiretroviral treatment prior to pregnancy

HIV-infected pregnant women who have CD4 count $\geq 250$ cells $/ \mathrm{mm}^{3}$ may have a higher chance of developing NVP-associated hepatotoxicity than those who have CD4 count $<250$ cells $/ \mathrm{mm}^{3}$ [12-16]. For practical reasons, a lopinavir/ritonavir (LPV/r)-based HAART is therefore recommended for all HIVinfected pregnant women who have not been on HAART prior to pregnancy and who will start HAART during pregnancy.

HIV-infected pregnant women who present to antenatal care prior to 14 weeks of gestation should have CD4 count available before considering an appropriate time for HAART initiation. However, an LPV/r-based HAART should be initiated at the first antenatal care visit for those who present to antenatal care after 14 weeks of gestation or for those who are symptomatic, without the need to wait for CD4 count result. In such cases, initiating HAART at the first antenatal care visit helps ensure that HIV-infected pregnant women are not missing the opportunity to receive PMTCT during early pregnancy or become lost to follow-up from the antenatal care system without PMTCT knowledge. HAART initiation during 14-24 weeks of gestation will provide adequate duration (at least three months) for ARV drugs to maximally suppress plasma HIV-1 RNA to the lowest level before delivery for the reduction of HIV transmission to infants.

Table 2 shows recommended HAART regimen and time to initiate and discontinue ARV drugs in HIVinfected pregnant women who have not been on antiretroviral treatment prior to pregnancy.

If HIV-infected pregnant women develop side effects from the use of ARV drugs or cannot tolerate ARV drugs, the following recommendations can be considered:

- If the woman cannot tolerate LPV/r due to severe diarrhea, LPV/r should be replaced with efavirenz (EFV) $600 \mathrm{mg}$ every 24 hours. EFV use is contraindicated during the first trimester.

- If the woman cannot tolerate AZT due to severe anemia, AZT should be replaced with stavudine (d4T) 30 mg every 12 hours.

Table 2. HAART regimen and timing for initiation and discontinuation for HIV-infected pregnant women who have not been on antiretroviral treatment prior to pregnancy.

\begin{tabular}{|c|c|c|c|}
\hline \multirow{2}{*}{$\begin{array}{l}\text { CD4 count } \\
\left(\text { cells } / \mathbf{m m}^{3}\right)\end{array}$} & \multirow{2}{*}{$\begin{array}{c}\text { First-line recommended } \\
\text { HAART }{ }^{* *}\end{array}$} & \multicolumn{2}{|c|}{ Timing of HAART regimen } \\
\hline & & Initiation $^{\#}$ & Discontinuation \\
\hline$\leq 350^{*}$ & $\begin{array}{l}\text { AZT + 3TC every } 12 \text { hours } \\
+ \text { LPV/r } 2 \text { tablets every } 12 \text { hours }\end{array}$ & As early as possible & $\begin{array}{l}\text { Continue HAART after delivery } \\
\text { Refer for follow-up care with } \\
\text { the internists and regimen } \\
\text { modification according to the } \\
\text { National Adult HIV Treatment } \\
\text { and Care Guidelines }\end{array}$ \\
\hline$>350$ & $\begin{array}{l}\text { AZT + 3TC every } 12 \text { hours } \\
+ \text { LPV/r } 2 \text { tablets every } 12 \text { hours }\end{array}$ & $\begin{array}{l}\text { As early as possible after } 14 \\
\text { weeks of gestation }\end{array}$ & $\begin{array}{l}\text { Discontinue all ARV drugs } \\
\text { together after delivery }\end{array}$ \\
\hline
\end{tabular}

* HIV-infected pregnant women who have CD4 count $>250$ cells $/ \mathrm{mm}^{3}$ may have a higher chance of developing NVPassociated hepatotoxicity than those who have CD4 $<250 \mathrm{cells} / \mathrm{mm}^{3}[12-16]$. LPV/r-based ARV regimen is therefore recommended for all HIV-infected women who will start HAART during pregnancy for practical purposes. **AZT 200-300 mg every 12 hours, 3TC $150 \mathrm{mg}$ every 12 hours, LPV/r (200/50 mg tablet) 400/100 mg every 12 hours. Dose adjustment of $\mathrm{LPV} / \mathrm{r}$ is not needed for HIV-infected Thai pregnant women during the third trimester [23, 24]. ${ }^{*} \mathrm{HIV}$-infected pregnant women who present to antenatal care prior to 14 weeks of gestation should have CD4 count available before considering appropriate time for ARV initiation. ARV regimen should be initiated at the first antenatal care visit for those who present to antenatal care after 14 weeks of gestation as LPV/r-based ARV regimen will be used regardless of CD4 count.

HAART = highly active antiretroviral therapy, AZT = zidovudine, 3TC = lamivudine, LPV/r $=$ lopinavir/ritonavir, ARV = antiretroviral. 
- If the woman cannot tolerate both LPV/r and EFV, or if the woman refuses to use a HAART regimen, an HIV specialist should be consulted. AZT monotherapy should be given from 14 weeks of gestation at the minimum. In this case, intrapartum SD-NVP is needed along with the provision of AZT + 3TC for seven days after delivery to reduce the development of NVP resistance.

\section{Scenario 2: HIV-infected pregnant women who conceive while on HAART}

HAART should be continued in HIV-infected pregnant women who conceive while on HAART for their own health. The efficacy of the current HAART regimen should be urgently assured from CD4 count and plasma HIV-1 RNA level. The best HAART regimen is the regimen that provides an undetectable plasma HIV-1 RNA level. An HIV specialist should be consulted if treatment failure is suspected from detectable plasma HIV-1 RNA level, especially if plasma HIV-1 RNA level is $\geq 1,000$ copies/mL, while the woman has adhered to the current HAART regimen for more than six months.

If the current HAART regimen is successful, consider carefully whether ARV modification is needed. If the current HAART regimen does not contain AZT, replace one of the nucleoside reverse transcriptase inhibitors used with AZT except if the woman previously experienced AZT resistance or severe side effects from AZT, such as anemia. If the current HAART regimen contains EFV and the woman is still in the first trimester of her pregnancy, EFV should be replaced with LPV/r. Use of EFV during the first trimester is not an indication for termination of pregnancy.

\section{Intrapartum antiretroviral drugs}

Antepartum HAART regimen must be continued during the intrapartum period. Oral AZT 300 mg every three hours or oral AZT $600 \mathrm{mg}$ given as a single dose at onset of labor is added regardless of antepartum HAART regimen or the woman's history of AZT resistance. The purpose of giving intrapartum AZT is to ensure adequate AZT levels in infants to prevent HIV acquisition during delivery, as the transmitting virus may be the wild type that is susceptible to AZT. Moreover, AZT passes to newborn babies rapidly and efficiently, and penetrates well to the central nervous system where HIV can reside long-term. SD-NVP is only given to HIV-infected pregnant women who need to use AZT monotherapy during the antepartum period [8]. Use of methergine should be avoided in HIV-infected pregnant women who are taking LPV/r or EFV due to the possible development of severe vasoconstriction. Oxytocin can be used instead.

\section{Postpartum antiretroviral drugs for mothers and infants}

Antepartum CD4 count is used to consider the continuation or discontinuation of ARV regimen in mothers after delivery (see Table $\mathbf{1}$ and 2).

AZT syrup $4 \mathrm{mg} / \mathrm{kg} /$ dose every 12 hours must be given to infants as soon as possible after delivery for four weeks. SD-NVP is only provided to infants born to mothers who received intrapartum SD-NVP. For infants born at less than 30 weeks of gestation, AZT syrup is given at $2 \mathrm{mg} / \mathrm{kg} /$ dose every 12 hours for four weeks. For infants born at 30-35 weeks of gestation, AZT syrup is given at $2 \mathrm{mg} / \mathrm{kg} /$ dose every 12 hours for two weeks then at $3 \mathrm{mg} / \mathrm{kg} /$ dose every 12 hours for two weeks [17].

Management of HIV-infected pregnant women who present in labor without antenatal care, and their infants (no ANC)

In HIV-infected pregnant women who do not receive any antepartum ARV drugs, the chance of transmitting HIV infection to their infants is as high as $20-40 \%$. ARV drugs should be given immediately to women who first present in labor, aiming to prepare adequate drug levels in their newborn infants during delivery. If the progression of labor is not rapid, caesarian section performed two hours post ARV drugs may help reduce HIV transmission.

\section{Antiretroviral drugs for $\mathrm{HIV}$-infected pregnant women who present in labor without antenatal care}

SD-NVP 200 mg must be given immediately along with oral AZT 300 mg every three hours until delivery or oral AZT $600 \mathrm{mg}$ given as a single dose at onset of labor. AZT + 3TC + LPV/r should be given after delivery until the results of CD4 count become available. If CD4 count is $>350$ cells $/ \mathrm{mm}^{3}$, a fourweek course of AZT + 3TC + LPV/r should be completed before all drugs are discontinued together. This is to reduce the development of NVP resistance [18], as this group of women can have a very high plasma HIV-1 RNA level which can increase the chance of developing NVP resistance. If CD4 count 
is $\leq 350$ cells $/ \mathrm{mm}^{3}$, the HAART regimen should be modified according to the National Adult HIV Treatment and Care Guidelines, i.e. to replace LPV/ $r$ with $\mathrm{EFV}$ along with the provision of family planning counseling.

If delivery is expected within two hours of presentation, SD-NVP should not be given to these women as there is not adequate time for NVP to cross the placenta to their infants. Furthermore, SD-NVP can unnecessarily create the development of NVP resistance in these women. Oral AZT 300 mg every three hours until delivery or AZT $600 \mathrm{mg}$ as a single dose should be given. AZT can be discontinued after delivery. HAART regimen should not be initiated intrapartum. The decision to initiate ARV regimen if CD4 count is $\leq 350$ cells $/ \mathrm{mm}^{3}$ should follow those recommendations in the National Adult HIV Treatment and Care Guidelines.

\section{Antiretroviral drugs for infants born to HIV-} infected pregnant women who did not receive antenatal care

ARV drugs should be immediately given to infants born to HIV-infected pregnant women who did not receive antenatal care. NVP syrup $2 \mathrm{mg} / \mathrm{kg}$ should be given every 24 hours for seven days followed by NVP syrup $4 \mathrm{mg} / \mathrm{kg}$ every 24 hours for seven days (total NVP duration is two weeks). AZT syrup $4 \mathrm{mg} /$ $\mathrm{kg} /$ dose should also be given every 12 hours with 3TC syrup $2 \mathrm{mg} / \mathrm{kg} /$ dose every 12 hours for four weeks [19]. Some experts recommend extending NVP for another two weeks at the dose of $4 \mathrm{mg} / \mathrm{kg}$ every 12 hours in week 3 and 4 of life (total NVP duration is four weeks), and extend AZT + 3TC to six weeks. The reason for this recommendation is to allow the preliminary diagnosis of HIV infection by PCR before the decision to discontinue ARV drugs in these infants is made.

In infants aged less than 48 hours who have not yet received any ARV drug after delivery, ARV drugs should be initiated immediately as described above. However, it is not recommended to initiate ARV drugs in infants who are more than 48 hours old, who have not yet received any ARV drugs after delivery, as it is unlikely that ARV drugs will provide any benefit [20]. These infants should be followed up closely as there is a high chance of HIV acquisition.

\section{Laboratory monitoring of $\mathrm{HIV}$-infected pregnant women receiving HAART}

Table 3 details the laboratory monitoring required in all HIV-infected pregnant women who receive HAART. LPV/r can cause metabolic side effects, including increased fasting plasma glucose level, in HIV-infected adults. Whether the use of LPV/r-based HAART increases the chance of pregnant women developing gestational diabetes is inconclusive [21, 22]. NNRTI may cause hepatitis, which can be serious in pregnant women. HIV-infected pregnant women require close clinical and laboratory monitoring and appropriate management.

\section{Mode of delivery Vaginal delivery}

Invasive procedures e.g. fetal scalp electrodes, forceps extraction, vacuum extraction, and artificial rupture of the membrane should be avoided unless medically indicated. The risk of MTCT is increased with premature rupture of the membrane (PROM) > four hours prior to delivery. In case of PROM, labor should be induced to reduce the time to delivery. Episiotomy should be performed carefully at the appropriate time to reduce exposure of the neonates to maternal blood and secretions.

\section{Elective caesarean section}

Elective cesarean section before the onset of labor and before the rupture of membrane can reduce the risk of MTCT. However, the risk of maternal complications is higher than in normal deliveries. In settings where cesarean section is feasible and safe, elective cesarean section should be considered in HIVinfected pregnant women at 38 weeks of gestation especially for those who did not receive antepartum HAART, received HAART for less than four weeks before the onset of labor, had poor ARV adherence, or had HIV RNA levels (if available) at 36 weeks of gestation $>1,000$ copies/mL. AZT $300 \mathrm{mg}$ every three hours for at least two doses or AZT $600 \mathrm{mg}$ given as a single dose four hours before the elective caesarean section should be given.

\section{Management in special situations \\ Pregnant women who are failing the HAART regimen due to drug resistance}

Pregnant women who have evidence of resistance to ARV components of the recommended HAART regimen should receive expert consultation for appropriate selection of an alternative HAART regimen. HAART given during pregnancy aims for 
Table 3. Recommended laboratory monitoring of HIV-infected pregnant women receiving HAART regimen.

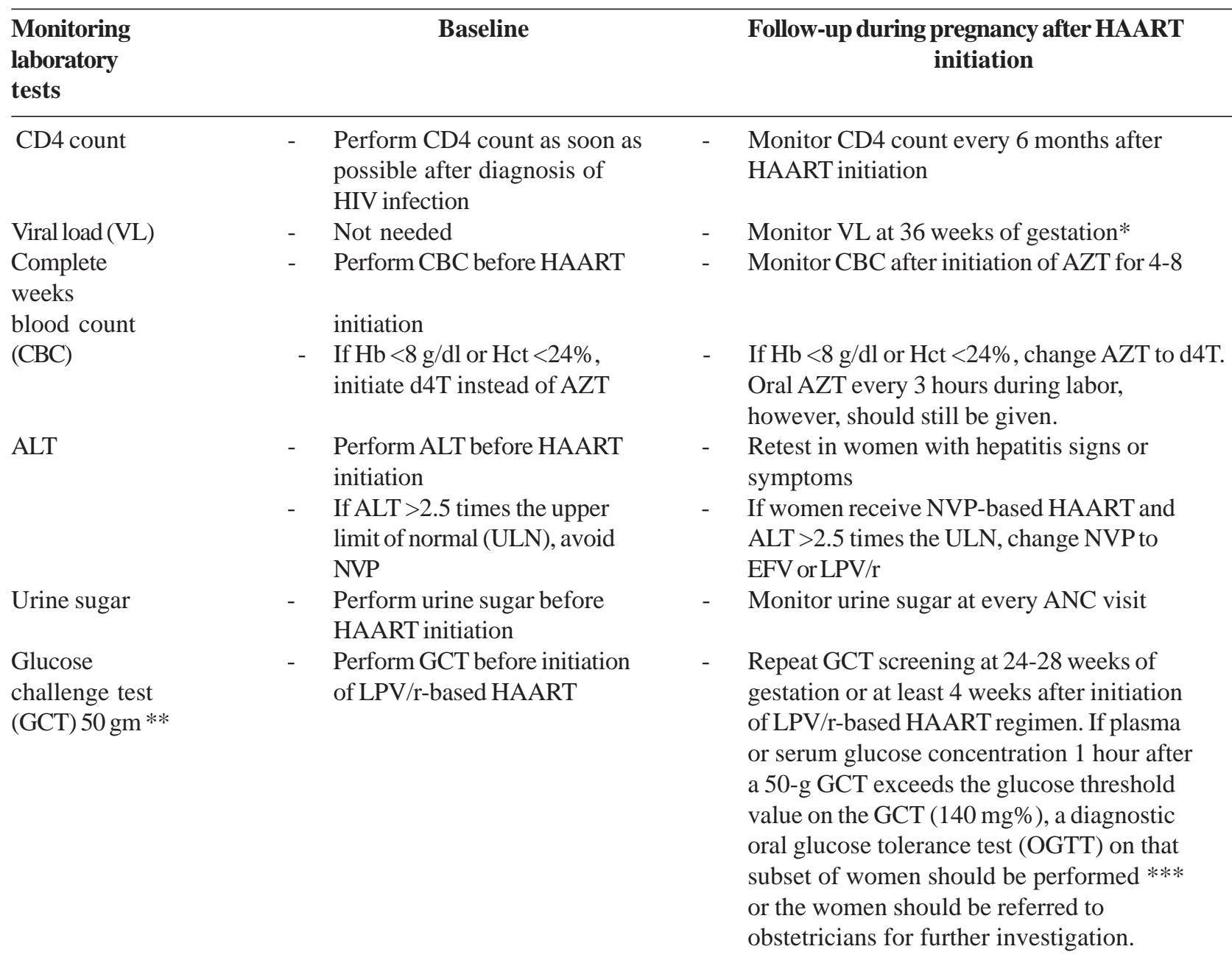

Pregnant women who have positive HBsAg should be referred to internists for consultation before discontinuation of HAART postpartum. * VL should be performed at least once at 36 weeks of gestation. ** GCT (Glucose challenge test) 50 gm: measuring the plasma or serum glucose concentration 1 hour after a 50-g oral glucose load. Evidences are inconclusive if LPV/r-based HAART regimen can increase a women's risk of developing gestational diabetes mellitus (GDM). Women receiving LPV/r-based HAART should undergo GCT as soon as feasible regardless of clinical characteristics consistent with a high risk of GDM (marked obesity, personal history of GDM, glycosuria, or a strong family history of diabetes). If they are found not to have GDM at that initial screening, they should be retested between 24 and 28 weeks of gestation. ***OGTT (Oral glucose tolerance test): measuring the fasting plasma or serum glucose concentration at 1, 2, and 3 hours after a 100-g oral glucose load. All women with fasting blood glucose concentration exceeds the glucose threshold value should be referred to obstetricians for proper management.

HAART = highly active antiretroviral therapy, VL = viral load, CBC = complete blood count, Hb = hemoglobin, Hct = hematocrit, $\mathrm{AZT}=$ zidovudine, $\mathrm{d} 4 \mathrm{~T}=$ stavudine, $\mathrm{ALT}=$ alanine aminotransferase, $\mathrm{ULN}=$ upper limit of normal, $\mathrm{NVP}=$ nevirapine, $\mathrm{EFV}=$ efavirenz, LPV/r = lopinavir/ritonavir, ANC = antenatal care, GCT = glucose challenge test, OGTT = oral glucose tolerance test.

complete viral suppression, the most important factor to prevent MTCT. Regardless of the HAART regimen during pregnancy, these women should receive AZT 300 mg every three hours during labor even if there is evidence of AZT resistance. Intrapartum AZT will be transferred to the infants and may help prevent perinatal HIV infection which is mostly caused by wild type virus. 
Treatment regimen for pregnant women who may have poor adherence

Women who have evidence of difficulties in adhering to HAART, despite adherence assistance and counseling, should be offered a HAART regimen with fewer number of pills such as AZT + 3TC + $\mathrm{EFV}$, starting after the first trimester. Although it has lower efficacy, treatment with AZT monotherapy starting at 14 weeks of gestation with SD-NVP during labor [8] may also be considered in women with CD4 count $>350$ cells $/ \mathrm{mm}^{3}$.

Pregnant women who received less than 4 weeks of HAART before delivery or had treatment interruptions or poor adherence during pregnancy

Elective caesarean section should be considered to such cases. The infants should receive HAART similar to that recommended in infants whose mothers are without antenatal treatment (no ANC), i.e., AZT $+3 T C+$ NVP for two to four weeks, then AZT + 3TC for another two weeks.

Pregnant women who are seronegative during the antenatal period, but whose partners are infected with HIV

If the women had no sexual exposure in the past three months, they must be counseled for consistent condom use. HIV serology should be tested again at 36 weeks of gestation.

If the women had recent sexual exposure, therefore at risk of being in the "window period", the appropriate test for viral antigen such as HIV DNA PCR or RNA PCR qualitative assay or HIV RNA PCR quantitative assay should be performed immediately.

Women considered being at high risk of recent HIV infection, although unproven, who present in labor or within two days after delivery should receive intrapartum and post partum management similar to HIV-infected pregnant women who have not received antenatal treatment (no ANC). The infants born to these women should receive HAART similar to that recommended in infants whose mothers are without antenatal treatment (no ANC). Postpartum HIV serology must be performed to confirm HIV infection in the women.

Pregnant women with indeterminate HIV serology These women must be tested with HIV DNA PCR or RNA PCR qualitative assay or HIV RNA
PCR quantitative assay immediately in order to initiate the appropriate treatment without delay, and maximize the efficacy of treatment to prevent MTCT.

\section{Pregnant women who received SD-NVP in the previous pregnancy}

These women should receive the regimen of $\mathrm{AZT}+3 \mathrm{TC}+\mathrm{LPV} / \mathrm{r}$ regardless of CD4 count due to the significant risk of NNRTI resistance. Recommendations in Table 1 and 2 should be followed.

Recommendations for the postpartum management of infants born to HIV-infected women

- Wear gloves while exposed to blood or body secretions.

- Clamp and cut umbilical cord carefully to reduce blood splash contamination.

- Dry and clean infant's skin with a warm cloth to reduce contamination with maternal blood or secretions before transferring to the nursery.

- Avoid unnecessary use of gastric tube to prevent mucosal trauma.

- Give infant formula and completely avoid breastfeeding or mixed feeding.

- $\quad$ Start ARV drug(s) (see Table 1 and 2).

- Give vitamin K and routine vaccinations for infants, including BCG vaccine and $\mathrm{HBV}$ vaccine.

\section{Comprehensive care for HIV-infected women} and family during the postpartum period

The goals of the program are to reduce MTCT, provide health promotion for infants born to HIVinfected mothers, and provide appropriate medical treatment for parents in order to reduce the risk of infants or children being orphaned. Comprehensive care for HIV-infected women and family includes the following services:

\section{Medical care during the postpartum period}

1) Standard postpartum care should be provided. Attention should be paid to specific postpartum issues, e.g. puerperal infection, side effects from ARV drugs, provision of medication to inhibit lactation and prevent breast engorgement or mastitis, and postpartum check up at four to six weeks after delivery, including cervical Pap smear. HIV-infected women are recommended to undergo cervical Pap smear screening for cervical cancer annually. 
2) General health promotion, e.g. nutritional support and exercise, should also be provided.

3) All postpartum women should be referred to internists for standard HIV treatment and care.

\section{Psychological management}

Psychological and social supports needed for HIVinfected families may include the management of postpartum depression, psychosocial support for child rearing, and long-term family care.

\section{Caring for male partner}

If the male partner is unaware of the woman's HIV status, readiness for disclosure of HIV status to the male partner should be assessed and promoted. Voluntary HIV counseling and testing should be offered for partners of HIV-infected women. HIV-infected male partners should be referred for standard HIV treatment and care. Male partners of HIV-infected women should receive positive health promotion services including:

- Promotion of safer sex practices, e.g. consistent condom use, sexual activities without insertion, and reducing risk behaviors for HIV acquisition.

- Promotion of general health care, e.g. good nutrition, exercise, and avoidance of substance abuse

- Advice on how to live happily with an HIV-infected partner.

- HIV-negative male partners should have repeat HIV testing every six months.

\section{Family planning services and contraceptive counseling}

The aim of family planning is to prevent unintended pregnancy and HIV transmission to HIVuninfected partner. The recommendation is to assess future pregnancy wishes in HIV-infected women and partners and provide family planning services.

1) HIV-infected women and partners planning to have children should receive pre-conceptual counseling on MTCT risks, their long-term health, and possible effects of ARV drugs on the fetus. Couples should carefully weigh risks and benefits. Couples who decide to have children should be advised on ways to reduce risk of HIV transmission to infants and partners as follows:

- HIV discordant couples in which the woman is infected with HIV should be referred to an obstetrician for intrauterine insemination technique to prevent HIV transmission to the male partner.
- HIV discordant couples in which the man is infected with HIV should be referred to an obstetrician for sperm wash and assisted conception technique to prevent HIV transmission to the female partner.

- HIV concordant couple who have full virological suppression through long-term HAART may be advised to have unprotected sexual intercourse at the time of ovulation.

2) HIV-infected women and partners planning not to have children should receive appropriate contraception counseling using "dual methods of contraception" which include consistent condom use plus other birth control options, e.g. sterilization (for those who do not want any more children), hormonal implantation, hormonal injection or intrauterine device (for those who may want to have children in the future). Women should be informed about drug interactions between oral contraceptive pills and antiretroviral drugs, e.g. NVP or some protease inhibitor drugs that may reduce the efficacy of birth control pills.

\section{HIV diagnosis for infants born to HIV-infected mothers and comprehensive care for HIV-infected infants}

1) ARV drugs for infants need to be provided as recommended in Table 1.

2) HIV-infected mothers need to receive counseling on infant formula feeding. Infant formula is provided without charge for 18 months by Thai MOPH.

3) Infants need to be assessed for signs and symptoms of HIV infection and side effects from ARV drug(s).

4) Infants need to receive appropriate vaccination.

5) Initiate cotrimoxazole (trimethoprimsulfamethoxazole, TMP-SMX) prophylaxis for Pneumocystis jiroveci pneumonia (TMP $150 \mathrm{mg} / \mathrm{m}^{2}$ of body surface area/day and SMX $750 \mathrm{mg} / \mathrm{m}^{2}$ of body surface area/day) three times per week beginning at four to six weeks of age and continue until HIVinfection is excluded or until infants are aged more than six months without HIV clinical symptoms. HIVinfected infants should be given prophylaxis until one year of age, at which time reassessment is made based on the age-specific CD4 count or percentage thresholds according to the National Pediatric HIV Treatment and Care Guidelines.

6) Laboratory tests to determine HIV status of the infants include HIV DNA PCR and HIV antibody test. 
- HIV DNA PCR should be performed at one to two months of age and at two to four months of age. If the first HIV DNA PCR is positive, a confirmation test should be done as soon as possible. If the first HIV DNA PCR is negative, the test should be repeated after four months of age.

- HIV antibody test should be performed at 12-18 months of age.

- HIV-infected infants should be referred to pediatricians for appropriate care.

\section{Acknowledgement}

The PMTCT Committee of the Thai National HIV Guidelines Working Group acknowledges the very active participation of the following members:

The PMTCT Committee of the Thai National HIV Guidelines Working Group acknowledges the very active participation of the following members:

Achara Teeraratkul, Amphan Chalermchockcharoenkit, Anan Muangmoonchai, Aram Limtrakul, Aree Kumpitak, Kulkanya Chokephaibulkit, Manoon Leechawengwong, Michelle McConnell, Nantawan Kaewpoonsri, Narisa Mantharngkul, Nipunporn Voramongkol, Nittaya Phanuphak, Peeramon Ningsanond. Piyawan Limpanyalert, Praphan Phanuphak, Rangsima Lolekha, Sanchai Chasombat, Sarawut Boonsuk. Siriporn Kanchana, Sombat Thanprasertsuk, Somnuek Sungkanuparph, Surasith Chaithongwongwatthana, Taweesap Siraprapasiri, Thananda Naiwatanakul, Wasun Chantratita, Wichai Techasathit

\section{References}

1. Department of Health. Guidelines of prevention of mother-to-child HIV transmission management and care for HIV-infected women and families. Department of Health, Ministry of Public Health, May 2007.

2. McIntyre JA, Martinson N, Gray GE, for the Trial 1413 Investigator Team. Single dose nevirapine combined with a short course of combivir for prevention of mother to child transmission of HIV-1 can significantly decrease the subsequent development of maternal and infant resistant virus. Antivir Ther. 2005; 10 (suppl 1): S4.

3. Results of HIV Sero-surveillance, Thailand 1989-2008. Source: AIDS division, Bureau of Epidemiology, Department of Disease Control. Available at http:// epid.moph.go.th/.

4. Naiwatanakul T, Punsuwan N, Kullerk N, Faikratok W, Lolekha R, Sangwanloy O, et al. Reduction in HIV transmission risk following recommendations for CD4 testing to guide selection of prevention of mother-tochild (PMTCT) regimens, Thailand, 2006-2007. Presented at 5th International AIDS Society Conference on HIV Pathogenesis and Treatment. Capetown, 19-22 July 2009 (Abstract CDC019).

5. Health promotion project evaluation report in social and economic development agenda 9. (February 2007). Business printing office of veteran support organization publisher.

6. Ad hoc reporting of Saiyairak family project. Department of Health. Available at http://www. saiyairakhospital.com/newdemo/admin/user_report. html, Accessed Dec 23, 09.

7. Bureau of Health Promotion, Department of Health, Bureau of Epidemiology, Department of Disease Control, and Thailand MOPH-U.S. CDC collaboration. Presented at Meeting to report the National PMTCT Program Evaluation 2007, Bangkok, 2 February 2008.

8. WHO. Rapid advice. Use of antiretroviral drugs for treating pregnant women and preventing HIV infection in infants. November 2009. http://www.who.int/hiv/ pub/mtct/advice/en/; Accessed 25 March, 2010.

9. Lallemant M, Jourdain G, Le Coeur S, Mary JY, NgoGiang-Huong N, Koetsawang S, et al. Single-dose perinatal nevirapine plus standard zidovudine to prevent mother-to-child transmission of HIV-1 in Thailand. N Engl J Med. 2004; 351:217-28.

10. Palombi L, Marazzi MC, Voetberg A, Magid NA. Treatment acceleration program and the experience of the DREAM program in prevention of mother-tochild transmission of HIV. AIDS. 2007; 21 (Suppl 4): S65-71.

11. Health Intervention and Technology Assessment Program (HITAP). Cost benefit and feasibility study of HAART for prevention of mother-to-child HIV transmission in Thailand. Presented at Steering Group Committee of HIV/AIDS System Development, Bangkok, November, 2009.

12. Timmermans S, Tempelman C, Godfried MH, Nellen J, Dieleman J, Sprenger H, et al. Nelfinavir and nevirapine side effects during pregnancy. AIDS. 2005; 19:795-9.

13. Jamisse L, Balkus J, Hitti J, Gloyd S, Manuel R, Osman N, et al. Antiretroviral-associated toxicity among HIV-1-seropositive pregnant women in Mozambique receiving nevirapine-based regimens. J Acquir Immune Defic Syndr. 2007; 44:371-6.

14. Marazzi MC, Germano P, Liotta G, Guidotti G, Loureiro S, da Cruz Gomes A, et al. Safety of nevirapinecontaining antiretroviral triple therapy regimens to 
prevent vertical transmission in an African cohort of HIV-1-infected pregnant women. HIV Med. 2006; 7: 338-44.

15. Kondo W, Carraro EA, Prandel E, Dias JM, Perini J, Macedo RL, et al. Nevirapine-induced side effects in pregnant women: experience of a Brazilian University Hospital. Braz J Infect Dis. 2007; 11:544-8.

16. Phanuphak N, Apornpong T, Teeratakulpisarn S, Chaithongwongwatthana S, Taweepolcharoen C, Mangclaviraj S, et al. Nevirapine-associated toxicity in HIV-infected Thai men and women, including pregnant women. HIV Med. 2007; 8:357-66.

17. Capparelli EV, Mirochnick M, Dankner WM, Blanchard S, Mofenson L, McSherry GD, et al. Pharmacokinetics and tolerance of zidovudine in preterm infants. J Pediatr. 2003; 142:47-52.

18. Russell VD, Jourdain G, Shapiro D, Ngo-Giang-Huong N, Frenkel L, Britto P, et al. A phase II study of the incidence of nevirapine resistance mutations in HIVinfected Thai women receiving a single intrapartum dose of NVP followed by a postpartum tail of ZDV/ddI or ZDV/ddI/LPV/r: IMPAACT P1032. Presented at 16th Conference on Retroviruses and Opportunistic Infections, Montreal, February, 2009 (Abstract 95aLB).

19. de Ruiter A, Mercey D, Anderson J, Chakraborty R, Clayden P, Foster G, et al. British HIV Association and Children's HIV Association guidelines for the management of HIV infection in pregnant women 2008. HIV Med. 2008; 9:452-502.
20. Wade NA, Birkhead GS, Warren BL, Charbonneau TT, French PT, Wang L, et al. Abbreviated regimens of zidovudine prophylaxis and perinatal transmission of the human immunodeficiency virus. N Engl J Med. 1998; 339:1409-14.

21. Gonzalez-Tome MI, Ramos Amador JT, Guillen S, Solis I, Fernandez-Ibieta M, Munoz E, et al. Gestational diabetes mellitus in a cohort of HIV-1 infected women. HIV Med. 2008; 9:868-74.

22. Hitti J, Andersen J, McComsey G, Liu T, Melvin A, Smith L, et al. Protease inhibitor-based antiretroviral therapy and glucose tolerance in pregnancy: AIDS Clinical Trials Group A5084. Am J Obstet Gynecol. 2007; 196:331 e1-7.

23. Ramautarsing $\mathrm{R}$, van der Lugt J, Phanuphak N, Gorowara M, Kerr S, Chuemchaitrakool A, et al. Standard dose generic lopinavir/ritonavir provides adequate lopinavir plasma levels during the 3rd trimester of pregnancy in Thai HIV-1 infected women. Presented at 11th Workshop on Clinical Pharmacology of HIV Therapy, Sorento, April, 2010 (Poster 9).

24. Cressy T, Jourdain G, Rawangban B, Yathavisuthi P, Varadisai S, Kongpanichkul R, et al, and the PHPT-5 Study Team. Pharmacokinetics of lopinavir boosted with ritonavir initiated during the third trimester of pregnancy in HIV-1-infected Thai women. Presented at 17th Conference on Retroviruses and Opportunistic Infections, San Francisco, February, 2010 (Abstract 906). 